\title{
Normal mode spectra of two-dimensional classical atoms confined by a Coulomb potential
}

\author{
W. P. Ferreira, ${ }^{1,2, *}$ F. M. Peeters, ${ }^{1, \dagger}$ and G. A. Farias ${ }^{2, *}$ \\ ${ }^{1}$ Departement Natuurkunde, Universiteit Antwerpen (Campus Drie Eiken), Universiteitsplein 1, B-2610 Antwerpen, Belgium \\ ${ }^{2}$ Departamento de Física, Universidade Federal do Ceará, Caixa Postal 6030, Campus do Pici, 60455-760 Fortaleza, Ceará, Brazil
}

(Received 9 July 2003; published 18 December 2003)

\begin{abstract}
The normal mode spectra of two-dimensional finite clusters of charged particles $(-e)$ confined by a Coulomb potential resulting from a displaced positive charge $Z e$ are obtained. This is a classical two-dimensional model system for atoms. We obtain the frequencies of the normal modes as a function of the confinement charge $Z$ and the number of particles $N$. The analysis of the lowest normal mode frequency reveals a good agreement with the experimental results obtained in a system with screened interaction between charged particles. The dependence of the normal mode spectra as a function of a perpendicular magnetic field is also discussed and we found that the shearlike character of the modes is enhanced in the presence of the magnetic field. For large values of the magnetic fields the normal modes fall into two bands, a low frequency band with frequency $\sim 1 / \omega_{c}$ and a high frequency band with frequency $\sim \omega_{c}$.
\end{abstract}

DOI: 10.1103/PhysRevE.68.066405

PACS number(s): 52.27.Lw, 36.40.Sx

\section{INTRODUCTION}

Recently, there has been an increased interest in the properties of two-dimensional (2D) systems consisting of a finite number of charged particles. These bounded clusters are kept together by an external field. Electrons in quantum dots [1], electrons on the surface of liquid helium [2,3], colloidal suspensions [4], and strongly coupled dusty plasmas [5] are some examples and experimental realizations of such $2 \mathrm{D}$ systems. From a theoretical point of view, this is a very interesting system which offers rich physics with several nontrivial effects (i.e., overcharging, phase transitions, Wigner crystallization), and in which the reduced dimensionality and the finite size of the system allow often an "exact numerical" description.

Most of the studies in such 2D systems were performed by considering the bare Coulomb interaction between the particles, e.g., see Refs. [6,7]. However, recently the 2D Debye-Hückel potential was also used as the interaction potential between particles in studying the structure, the melting, and the dynamical properties of dusty plasmas [8-11]. The exponentially screened interaction potential allowed a good agreement with the experimental results, mainly of the normal mode spectra [8]. It is important to emphasize that in all these cases, a parabolic confinement potential was used to keep the system bounded. This kind of confinement offers often a good approximation, but it can cover up some interesting characteristics of the system which depend on the strength of the confinement potential (in a parabolic confined system the strength of the potential can be scaled out [6]). Phase transition induced by evaporation of particles [12,13] is an example of such a property which depends on the strength and the kind of confinement potential, and which is not possible when using a parabolic potential (i.e., a Coulomb type of confinement potential which becomes zero at

\footnotetext{
*Electronic address: paiva@ fisica.ufc.br

†Electronic address: peeters@uia.ua.ac.be

*Electronic address: gil@ fisica.ufc.br
}

infinity). The possibility to tune the pair interaction potential or the confinement potential can reveal interesting and nontrivial behaviors in 2D ordered systems [14-17].

The dynamic properties of 2D classical finite systems are of fundamental interest. Instead of waves, in finite systems local normal modes are excited as a response to an external excitation. An experimental realizable system in which such normal modes in 2D clusters have been studied is the complex dusty plasma. Not like in most ordinary plasmas in space and laboratory, which are weakly coupled, a complex plasma is strongly coupled. It consists of many strongly charged dust particles immersed in a gaseous plasma rf discharge. Experimentally the shear and compressional character of the modes [8] have been investigated, and this is in agreement with the predicted theoretical results [7]. Very recently, one more ingredient has been added in the study of the normal modes in complex plasmas: an axial magnetic field. Depending on the discharge conditions, rigid-body rotation and sheared rotation are observed [18]. Similar behaviors of a dust cluster are observed in an inductively coupled rf plasma [19]. A coordinated study of a laboratory experiment found that the charged dust particles in a circle show angular rotation in which the structure stays intact, while they oscillate radially around the equilibrium orbit. This oscillatory rotational nature can be explained as a result of coupling between the Lorentz force and the harmonic confinement potential [20].

In the present paper, we consider a 2D classical system of negatively charged particles interacting through a pure Coulomb potential, and confined by a punctual positive charge. This system is very similar to the amply used 2D model of a dusty plasma with a parabolic confinement, but it presents the advantage to enable us to tune the strength of the confinement charge $Z$. In other words, we are considering a complex plasma in a nonuniform positive background, in contrast to the previous models which considered a uniform neutralizing positive background (the parabolic confinement potential corresponds to a uniform density of positive charges). Following the idea used in Ref. [14], changing $Z$ can work like a pseudotemperature, in the sense that it alters the inten- 


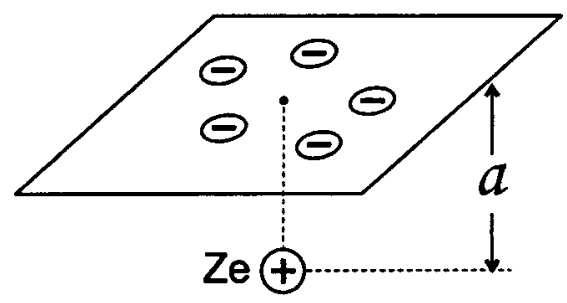

FIG. 1. Schematic view of the system.

sity of the pair interaction potential, which has a large influence on the structural and dynamical properties of the cluster. In a previous work [21] we investigated the ground state and the configurational properties of this system and indicated the importance of correlation effects. However, in this paper we mainly concentrate our attention on the behavior of the normal mode spectra, and its corresponding normal modes, as a function of $Z$ and $N$. The influence of a perpendicular magnetic field on the normal modes and eigenvectors is also studied.

This paper is organized as follows. In Sec. II we describe the numerical approach and mathematical model used to obtain the spectra. The results of the normal mode spectra and eigenvectors as a function of the confinement charge, the number of particles, and the magnetic field are presented in Sec. III. Our conclusions are given in Sec. IV.

\section{THE MODEL}

We study a system with $N$ negatively charged particles $(-e)$, which we call here and further electrons, interacting through a repulsive pure Coulomb potential and moving in the $x y$ plane. The particles are kept together through a fixed positive charge $Z e$ located at a distance $a$ from the plane the particles are moving in (see Fig. 1). The total energy of this Coulomb confined system is given by the Hamiltonian

$$
H=-\frac{Z e^{2}}{\epsilon} \sum_{i=1}^{N} \frac{1}{\sqrt{r_{i}^{2}+a^{2}}}+\frac{e^{2}}{\epsilon} \sum_{i>j=1}^{N} \frac{1}{\left|\vec{r}_{i}-\vec{r}_{j}\right|} .
$$

Here the symbol $\boldsymbol{\epsilon}$ stands for the dielectric constant and $\vec{r}$ $=\{x, y\}$ is the two-component position vector of the 2D electron. For convenience, we express the electron energy in units of $E_{0}=e^{2} / \epsilon a$ and all the distances in units of $a$. This allows us to rewrite Eq. (1) in the following dimensionless form:

$$
H=-\sum_{i=1}^{N} \frac{Z}{\sqrt{r_{i}^{2}+1}}+\sum_{i>j=1}^{N} \frac{1}{\left|\vec{r}_{i}-\vec{r}_{j}\right|}
$$

The ground state configurations of the two-dimensional system were obtained using the standard Metropolis algorithm (at zero temperature) and the modified Newton method $[7,22]$. The latter method was discussed in Ref. [7]. Depending on the number of particles $N$ and the confinement charge $Z$, different number of stable states can be obtained. For example, for a cluster with $N=70$ electrons and confined by a charge $Z=70$ we observed 16 stable configurations, while for the cluster with $N=19$ electrons, the maximum number of stable states was four, in the case of a confinement charge $Z=100$. In general, in order to have sufficient confidence that we reached the ground state configuration, we performed around 400 tests for each case, where we started each time with a different initial random configuration. However, for clusters with large $N$, a greater number of tests is necessary, mainly if we are close to the complete screened situation $(N \approx Z)$. As an example, for the cluster with $N=200$ electrons and confinement charge $Z=200$, we obtained 729 metastable states in 1000 tests. The difference in the energy between the ground state configuration, and the energy of the metastable configurations was within the range $10^{-5}-10^{-3}$. From all the obtained stable states, the one with the lower energy was taken as the ground state configuration. To check if a configuration is stable, we calculated, using the Householder diagonalization technique, the eigenvalues of the dynamical matrix [7]

$$
H_{\alpha \beta, i j}=\frac{\partial^{2} H}{\partial r_{\alpha, i} \partial r_{\beta, j}},
$$

which give us the square of the frequencies of the normal modes of the system $(\alpha, \beta=x, y$ and $i, j$ indicates the particle number). The configuration was taken as final when all frequencies of the normal modes were positive and real.

In order to compare the frequencies calculated in our Coulomb confined cluster with the ones for a different confinement potential (i.e., parabolic), the values of the frequencies were scaled by the factor

$$
\omega_{o}=\frac{\omega_{o}^{\prime}}{\sqrt{2}}=\sqrt{\frac{e^{2}}{m \epsilon a^{3}}} \sqrt{\frac{Z}{2}}
$$

with $m$ the mass of each particle. Equation (4) was obtained from a comparison between the scaling factor of the energy in the parabolic confined system [7] and the corresponding factor in the Hamiltonian of our Coulomb confined cluster in the limit $N / Z \ll 1$, in which case Eq. (2) may be expanded into

$$
H_{a p p r}=-\left(2 Z^{2}\right)^{1 / 3} N+\sum_{i=1}^{N} r_{i}^{2}+\sum_{i>j=1}^{N} \frac{1}{\left|\vec{r}_{i}-\vec{r}_{j}\right|},
$$

with the following scaling for the variables $H$ and $r$ :

$$
H \rightarrow(Z / 2)^{1 / 3} H, \quad \vec{r} \rightarrow(2 / Z)^{1 / 3} \vec{r}
$$

The energy in Eq. (5) is in unit of $E_{0}^{\prime}=\left(e^{2} / \epsilon a\right)(Z / 2)^{1 / 3}$. If we equal this scaling factor with the one which scales the energy in the parabolic confined system, $E_{0}$ $=\left(m \omega_{o}^{\prime 2} e^{4} / 2 \epsilon^{2}\right)^{1 / 3}$, we obtain Eq. (4).

\section{RESULTS AND DISCUSSIONS}

We will start first to discuss the general features of the normal mode spectrum for Coulomb clusters containing a small number of electrons. In particular, we consider the 


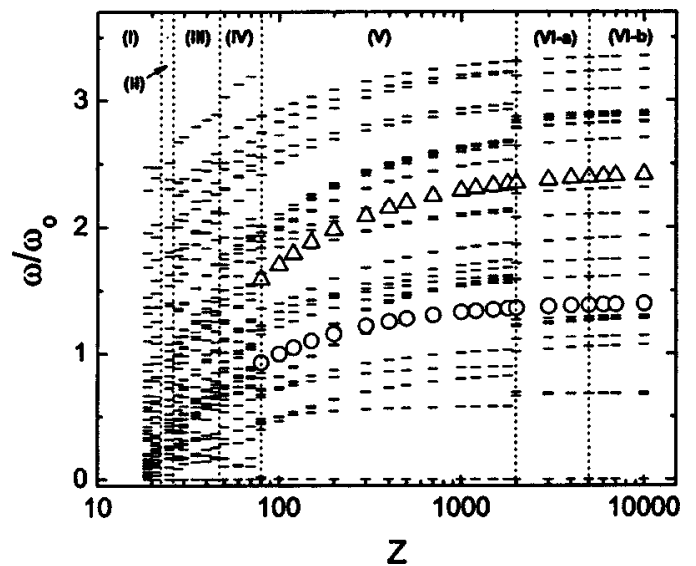

FIG. 2. (a) The eigenfrequencies for the $N=19$ cluster as a function of $Z$. The frequencies are in units of $\omega_{o}$. The six different regions [(I),(II),(III),(IV),(V),(VI-a,b)] indicate different ground state configurations. The frequencies of the center of mass (circles) and the breathing (triangles) modes are emphasized by different symbols.

cluster with $N=19$ electrons, which was extensively studied in the more simple case of parabolic confinement [7-9], and serves as a convenient case to emphasize particularities of the present system. We studied the properties of the normal mode spectrum as a function of the confinement potential or more precisely, the positive charge $Z$. A study of the nature of the excitations (shearlike or compressionlike) corresponding to the different normal modes will also be presented. Subsequently, we consider the behavior of the normal mode spectrum in the presence of a magnetic field. Different from the traditional approach of classical 2D systems, where a parabolic confinement potential is considered, the 2D classical system confined by a Coulomb potential has one extra parameter beyond the number of particles $N$, namely, the confinement charge $Z$. This feature allows us to tune the strength of the confinement potential which influences the ground state configurations [21] and the behavior of the normal modes. We will consider two regimes of confinement: (1) soft confinement (SFC), in which the number of particles $N$ is close to the value of the confinement charge $Z, N \approx Z$; and (2) strong confinement (STC), in which case the limit $N \ll Z$ is reached.

\section{A. Small clusters}

In Fig. 2 we present the frequencies of the normal modes as a function of $Z$ for a cluster with $N=19$ electrons. The frequencies were normalized by the factor $\omega_{o}$, as defined by Eq. (4). We observe a clear dependence of the distribution of modes for different $Z$ values. In the SFC regime most of the normal modes are concentrated around small frequencies, while in the STC regime the normal mode frequencies are more uniformly spread out over a larger frequency range. The distribution of normal mode frequencies is similar for all $N$ values, and it is better observed by defining the density of states (DOS). It is the number of frequencies in the interval $\delta \omega=\omega_{\max } / 40$, where $\omega_{\max }$ is the maximal frequency. The DOS for clusters with $N=19$ electrons, confined by charges
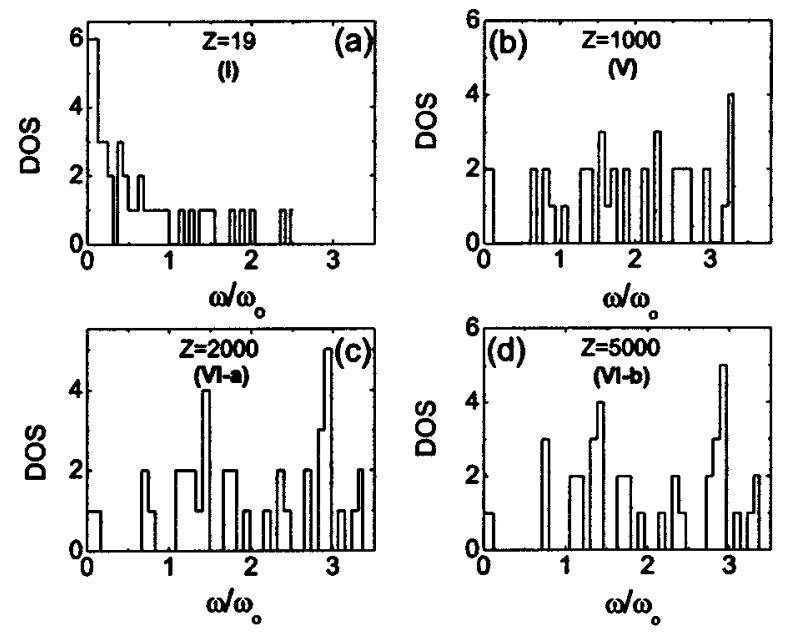

FIG. 3. The density of states (DOS) as a function of frequency for the $N=19$ cluster confined by a positive charge (a) $Z=19$, (b) $Z=1000$, (c) $Z=2000$, and (d) $Z=5000$.

$Z=19,1000,2000,5000$ as a function of the frequency, is shown in Figs. 3(a), 3(b), 3(c), and 3(d), respectively. In spite of the small number of particles, it is possible to identify the presence of two peaks $\left(\omega / \omega_{0} \approx 1.4\right.$ and $\omega / \omega_{0} \approx 2.9$ for $Z$ $=2000,5000)$ in the DOS for the cases $Z=2000$ and $Z$ $=5000$. The behavior shown in Figs. 3(c) and 3(d) is in qualitative agreement with previous results for a $2 \mathrm{D}$ classical infinite systems [23], where two types of waves, with dispersion relations $\omega \approx k$ (lateral sound waves) and $\omega \approx \sqrt{k}$ (longitudinal plasma waves) were observed. This last one arises from the long range nature of the Coulomb interactions [24]. These waves are related to the shearlike and compressionlike modes observed in our finite confined cluster and they will be discussed later in more detail.

In general, the spectrum moves to higher energies with increasing $Z$. With the exception of the trivial mode $\omega=0$ (i.e., uniform rotation of the system), no other frequency is independent of $Z$. The normal mode spectrum exhibits a discontinuous behavior as a function of $Z$, which is indicated in Fig. 2 by the six different regions [(I), (II), (III), (IV), (V), (VI-a,b)]. This is a consequence of structural phase transitions in the ground state configuration of the cluster. A more detailed description of these transitions and an analysis of the lowest normal modes will be given in the next paragraphs. We also emphasize in Fig. 2 the frequencies of the center of mass (circles) and the breathing (triangles) modes. The center of mass mode, as observed in a parabolic confined cluster, is a twofold degenerated vibration (with frequency $\omega=\sqrt{2}$ ) of all particles in the same direction and all of them moving in phase, while the breathing mode corresponds to a vibration of the mean squared radius of the cluster (with frequency $\omega=\sqrt{6}$ ). These special modes were found [7] to be independent of the number of particles and are a property of the particular form of the parabolic confinement. In our Coulomb confinement potential case, the observation of such modes is possible only in the limit of the STC regime [regions (V), (VI-a), and (VI-b) of Fig. 2]. However, we noticed that the frequencies of the center of mass mode become degenerate only in region (VI), where the ground state configu- 

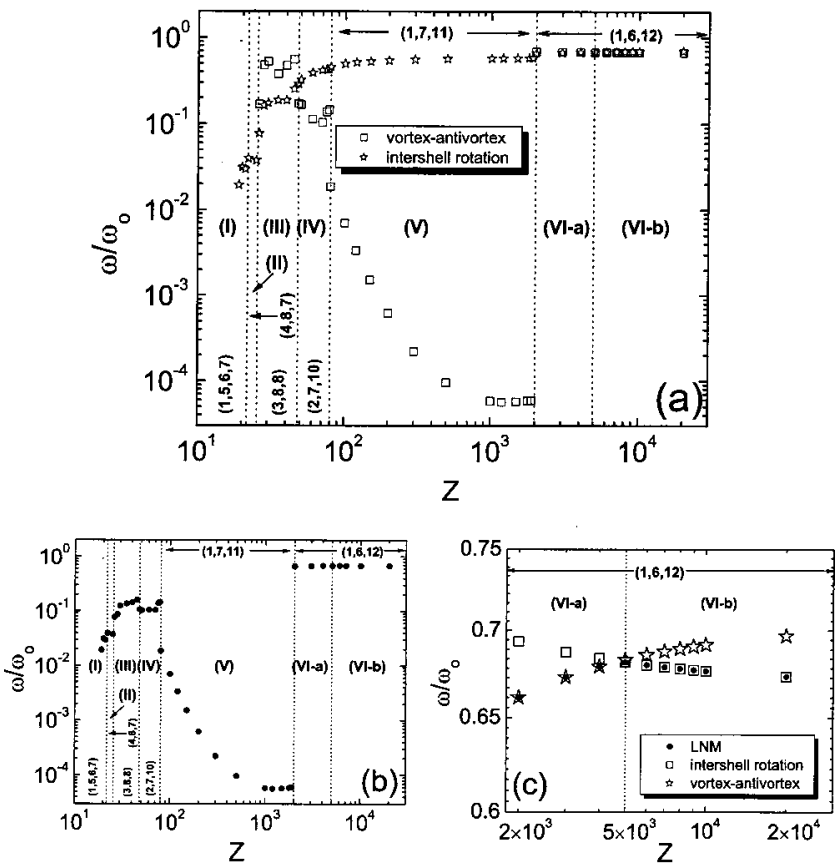

FIG. 4. (a) The frequencies of the intershell rotation mode (squares) and of the vortex-antivortex excitation (stars) as a function of $Z$ for the $N=19$ cluster. (b) The frequencies of the $L N M \mathrm{~s}$ as a function of $Z$ for the same cluster. (c) A more detailed view of region $(\mathrm{VI})$. The six regions were labeled $[(\mathrm{a}),(\mathrm{b})]$ in order to separate different ground state configurations, which are indicated in the figure.

ration of the cluster is $(1,6,12)$ and it coincides with that observed in the parabolic confinement case. The degeneracy of the frequency of the center of mass mode can serve as a good test that the cluster is in the parabolic confinement regime.

The lowest nonzero normal mode (LNM) frequency is associated with the stability of the ground state configuration. It tells us how easy or difficult the configuration may be deformed. As previously presented in Ref. [7] for the parabolic confinement case, the LNM corresponding to the intershell rotation (ISR) depends on the configuration of the cluster. Commensurate configurations are more stable against ISR, and present a high value for the activation frequency of this mode. In the opposite case, incommensurate configurations can be easily excited in the ISR mode and correspond to a very small frequency. In the $2 \mathrm{D}$ Coulomb confined cluster a similar dependence of the LNM frequency as a function of the configuration of the cluster was observed. Clusters with a commensurate configuration presented a high activation frequency for the ISR mode. However, the appearance of the ISR mode also depends on the strength of the confinement potential. As an example, the cluster with $N=12$ electrons and ground state configuration $(4,8)$ may be activated in two different LNMs. For $Z=45$ the $L N M$ is a vortexantivortex pair, while for $Z=48$ the corresponding LNM is an intershell rotation. In the 2D Coulomb confined cluster, the charge $Z$ plays the dominant role in determining the lowest normal mode. This is a particular feature of the Coulomb

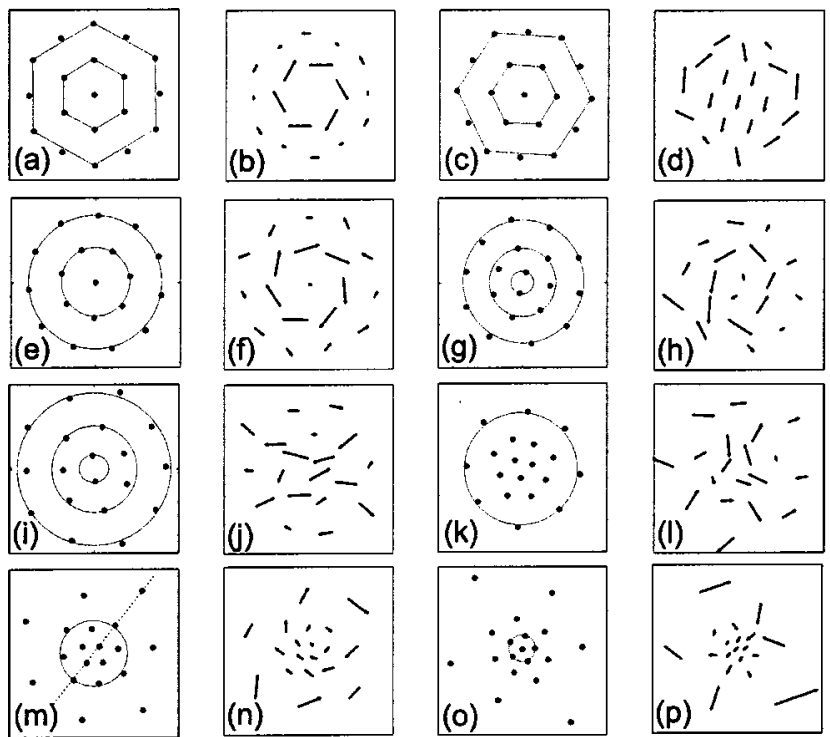

FIG. 5. The ground state configurations and the LNMs corresponding to the $N=19$ cluster with different confinement charges in the different regions shown in Fig. 4: $Z=5000$ [(a),(b)—region (VIb)]; $Z=2000[(\mathrm{c}),(\mathrm{d})$ —region $(\mathrm{VI}-\mathrm{a})] ; Z=500[(\mathrm{e}),(\mathrm{f})$ —region $(\mathrm{V})]$; $Z=75[(\mathrm{~g}),(\mathrm{h})$-region (IV)]; $Z=50[(\mathrm{i}),(\mathrm{j})$ —region (IV)]; $Z=30$ $[(\mathrm{k}),(\mathrm{l})$-region $\quad$ (III) $] ; \quad Z=22 \quad[(\mathrm{~m}),(\mathrm{n})$-region $\quad(\mathrm{II})] ; \quad Z=19$ $[(\mathrm{o}),(\mathrm{p})$ - region (I)]. The solid circles represent the charged particles in the $x y$ plane, while the arrows indicate the eigenvectors of the normal modes.

confinement potential, which is not observed in the case of parabolic confinement.

In order to identify the nature of the lowest normal mode as well as the importance of the parameter $Z$, we present in Fig. 4 the frequencies of the lowest normal mode, the frequency of the vortex-antivortex mode, and the frequency of the intershell rotation for each $Z$ value. More specifically, in Fig. 4(b) the frequencies of the lowest normal modes are shown as a function of $Z$ for a cluster with $N=19$ electrons. We also present in Fig. 4(a) the frequencies corresponding to the excitation of a vortex-antivortex pair (stars) and the intershell rotation mode (squares). Six regions were labeled in order to indicate the different ground state configurations, which are also indicated in the figures. For example, the configuration $(1,7,11)$ means one particle in the center, seven particles in first ring, and 11 particles in the second ring. We start our analysis in the limit of the STC regime, regions (V) and (VI). For $Z \geqslant 120$ only two stable configurations were obtained, namely, $(1,6,12)$ and $(1,7,11)$, and depending on the confinement charge $Z$ one of them corresponds to the ground state configuration. In region (VI-b), $Z \geqslant 5000$, the commensurate ground state configuration $(1,6,12)$ has the $L N M$ corresponding to the ISR [Figs. 5(a) and 5(b)]. This is the expected behavior, since in the limit $N \ll Z$ the confined Coulomb system behaves like a parabolic confined cluster [21]. The value of the LNM frequency is $\omega_{\min } / \omega_{0} \approx 0.681$, for $Z=5000$, which is very close to the observed value for the system with a parabolic confinement, $\omega_{\text {par }} / \omega_{0} \approx 0.668$ [7]. The vortex-antivortex pair excitation was observed with a frequency very close to the one for the ISR mode $\left(\omega / \omega_{0}\right.$ 
$\approx 0.683$ ). In region (VI-a), $2000 \leqslant Z<5000$, the ground state configuration is still $(1,6,12)$. However, a surprising change in the character of the LNM is observed [Fig. 4(c)]. The lowest normal mode becomes now a vortex-antivortex mode which has a smaller activation frequency [Figs. 5(c) and 5(d)]. This feature clearly illustrates that the LNM can be tuned by the strength of the confinement potential. A similar behavior was observed for different number of particles. It is also interesting to note that no significant modification is observed in the value of the LNM frequency when we change the value of $Z$ from region (VI-b) to region (VI-a), which has the same ground state configuration [see Fig. 4(c)]. The ISR mode is still observed in region (VI-a), but now with a slightly higher activation frequency. In Fig. 4(c) we emphasize the transition in the LNM frequency in the region (VI). In region (VI-b) the lowest normal mode frequency corresponds to the intershell rotation mode, while in region (VI-a) it is changed, corresponding now to a vortexantivortex pair.

For $80 \leqslant Z<2000$, region (V), the ground state configuration is $(1,7,11)$, and the configuration $(1,6,12)$ is now a metastable state. The LNM corresponding to the new ground state configuration is the ISR [Figs. 5(e) and 5(f)], and since the system presents an incommensurate configuration, the associated frequency is very small (for $Z=1900$ the LNM frequency is $\omega_{\min } / \omega_{0}=5.97 \times 10^{-5}$, while for $Z=80$ the $L N M$ frequency is $\left.\omega_{\min } / \omega_{0}=1.86 \times 10^{-2}\right)$. As shown in Fig. 4(b), there is a pronounced difference in the LNM frequency between regions $(\mathrm{V})$ and $(\mathrm{VI})$, which is associated with a structural phase transition in the cluster. It is worth to comment here that configurations as well as LNMs like those present in regions (V) and (VI) were recently observed in dusty plasma with a parabolic confinement [25], and in which the particles interact through a screened coulomb potential $[7,8,11]$.

The transition from region (V) to region (IV), $48 \leqslant Z$ $<80$, is characterized by a structural phase transition to the new ground state configuration $(2,7,10)$ [Fig. $5(\mathrm{~g})]$, and a jump in the value of the LNM frequency. The frequency of the ISR mode observed in region (IV) [Fig. 5(h)] is one order of magnitude larger than the one in region $(\mathrm{V})$. The increase in the LNM frequency is due to the formation of a new shell. The energy to activate the ISR mode in the configuration $(1,7,11)$ is lower than the necessary energy to activate the same mode in the configuration $(2,7,10)$. As a consequence, if we decrease the confinement strength $Z$, we still observe the configuration $(2,7,10)$ [Fig. 5(i)], but now with the LNM as shown in Fig. 5(j), which is similar in character to a vortex-antivortex pair. Once more, we noticed that for the same configuration different LNMs may be activated. With a further decrease of $Z$ the system exhibits a new structural transition to region (III), $26 \leqslant Z<48$, with ground state configuration $(3,8,8)$ [Fig. $5(\mathrm{k})]$. The system self-organizes into an incommensurate configuration with three shells. The corresponding LNM presented an unexpected asymmetry as shown in Fig. 5(1), which is a mixture of an intershell rotation and a vortex-antivortex pair excitation. Decreasing even further the value of $Z$, we reach region (II), $22 \leqslant Z<26$, where the ground state configuration is $(4,8,7)$ [Fig. $5(\mathrm{~m})$ ].

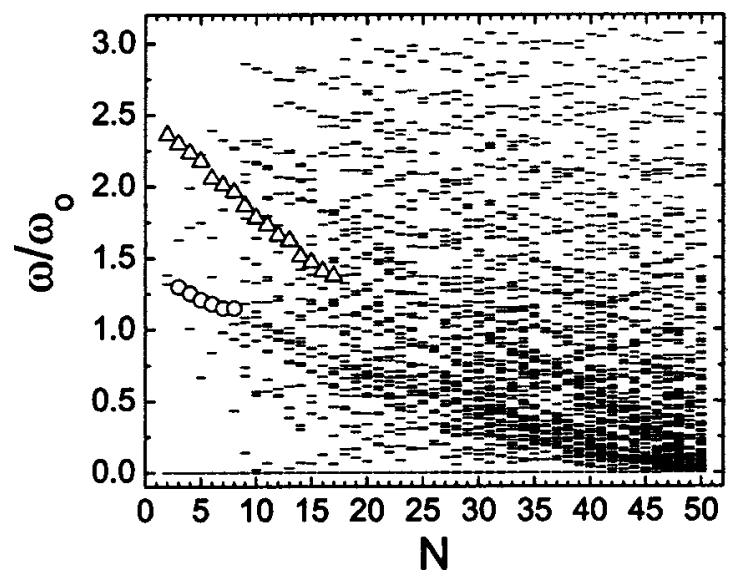

FIG. 6. Eigenfrequencies as a function of $N$. The confinement charge is $Z=50$. The frequencies are in units of $\omega_{0}$. The frequencies of the center of mass (circles) and the breathing (triangles) modes are emphasized by different symbols.

The LNM is an asymmetric vortex-antivortex pair [Fig. $5(\mathrm{n})]$. The entire cluster exhibits a mirror symmetry with respect to the dotted line in Fig. 5(m), but there is no rotational symmetry as in the STC regime. Finally for $Z \leqslant 22$, we are in the almost charge compensated case, region (I), with configuration $(1,5,7,6)$ [Fig. 5(o)]. The associated LNM is presented in Fig. 5(p), and it corresponds to a symmetric vortex-antivortex pair. A local circular symmetric configuration is observed for the most internal electrons. This reflects the symmetry of the confinement potential which acts more strongly at small distances. The effect of the confinement potential on the most external electrons is partially screened by the internal ones. As a consequence, the positional correlations between the electrons overcome the symmetry of the confinement potential. The maximum radius of the cluster in this region is one order of magnitude larger than the one in the STC regime. It is worth to comment that the ISR rotation mode was not observed in regions (I) and (II).

\section{B. Constant confinement and large clusters}

In this section we analyze the frequencies of the normal modes for clusters with different $N$, but submitted to a constant confinement potential (fixed $Z$ ). For this, we considered $Z=50$ and varied the number of particles from $N=2$ up to $N=50$. The main features of the ground state configurations in this case were previously presented in Ref. [21]. Here we limit ourselves to the results for the dynamical properties.

In Fig. 6 the frequencies of the normal modes are presented as a function of $N$. Only the frequency $\omega=0$, which represents a rotation of the entire cluster around the $z$ axis, is independent of $N$. An indication of the behavior of the center of mass (open circles) and the breathing (open triangles) modes as a function of the ratio $N / Z$ is also shown in Fig. 6. As observed before in Fig. 2, the frequencies of these modes decrease with increasing $N / Z$. The degenerate frequency of the center of mass mode is observed only in the clusters $N$ $=3,4,5,6,7,8$, and once more this corresponds to the same ground state configurations observed in the parabolic case. 

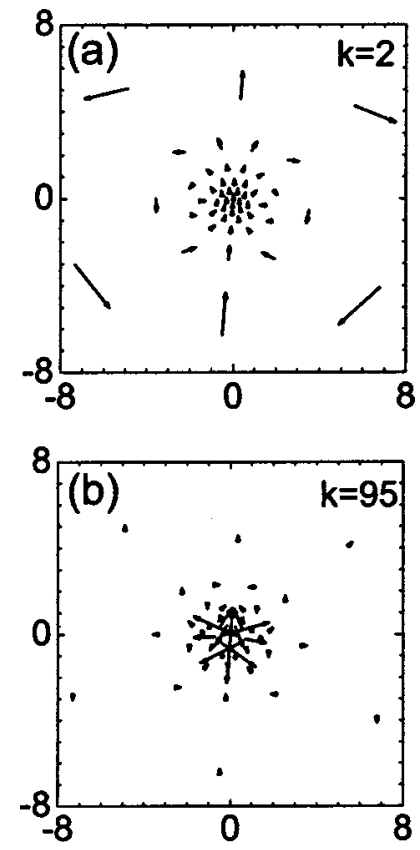

FIG. 7. Eigenvectors for the $N=50$ cluster confined by the positive charge $Z=50$. (a) the LNM $k=2$, and (b) the mode $k=95$.

Curiously the clusters $N=15$ and $N=16$ also present a degenerated-like center of mass (c.m.) mode, but the ground state configurations are different from the parabolic case. The main characteristic shown in Fig. (6) is again the high concentration of modes with small activation frequencies in the SFC regime, $N \approx Z$. We can understand this behavior taking the cluster $N=50(Z=50)$ as an example. In the SFC regime the maximum radius of the cluster is one order of magnitude larger than the one in the STC regime [26]. The large distance between the electrons localized at the border of the cluster makes the confinement of these particles very weak. This fact allows the excitation of modes with low frequencies. In fact, we observe that the external electrons are more affected in the low frequency modes, while the core electrons are practically frozen. This is shown in Fig. 7(a), where we plotted the eigenvectors for the LNM (mode number $k=2$, $\omega / \omega_{0}=3.574 \times 10^{-3}$ ) for a cluster with $N=Z=50$ electrons. In the high frequency region there is an inversion, and the core electrons are more affected than the external ones, as can be observed in Fig. 7(b) for a mode excited in the high frequency region (mode number $k=95, \omega / \omega_{0}=2.373$ ). In the limit $N / Z \ll 1$, the graphic of the density of states is similar to the one plotted in Fig. 3(d).

In general, we found that the relation $N / Z$ determines how the frequencies of the normal modes are distributed, and this is valid in a large interval of $N$ [27]. In order to show this behavior for large systems, we present in Figs. 8(a) and 8(b) the DOS for clusters with $N=200$ electrons, which are confined by positive charges $Z=200$ and $Z=4000$, respectively. As previously observed in small clusters, the DOS is large in the low frequencies region, when $N \approx Z$. In the STC regime, the appearance of two broad maxima is connected to shearlike and compression-like modes. This is demonstrated by calculating the spatial average of the divergence $(\vec{\nabla} \cdot \vec{A})$ and
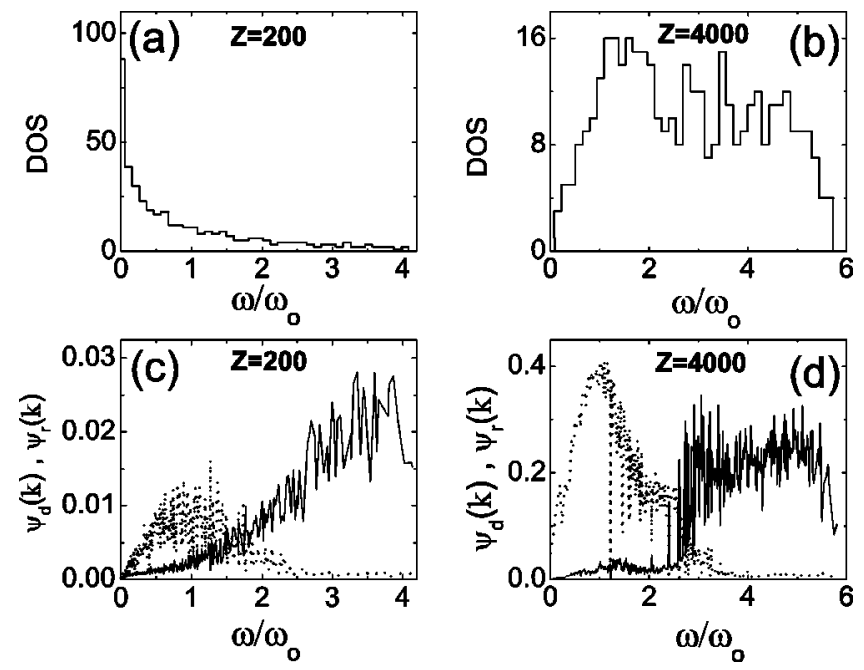

FIG. 8. The DOS as a function of frequency for clusters with $N=200$ and confinement charges (a) $Z=200$ and (b) $Z=4000$. The distribution of the rotor $\psi_{r}$ (dotted line) and the divergence $\psi_{d}$ (solid line) as a function of the normalized frequency for the same clusters $N=200$ : (c) $Z=200$ and (d) $Z=4000$.

the $z$ component of the vorticity $(\vec{\nabla} \times \vec{A})$ of the eigenvectors field, following the approach of Refs. $[7,11]$. The $z$ component of the rotor $\psi_{r}(k)=\vec{e}_{z} \cdot \operatorname{rot}[\psi(k)]$ and the divergence $\psi_{d}(k)=\operatorname{div}[\psi(k)]$ of the field of eigenvectors are

$$
\begin{aligned}
& \psi_{d}(k)=\frac{1}{N} \sum_{i=1}^{N} \psi_{d, i}^{2}(k), \\
& \psi_{r}(k)=\frac{1}{N} \sum_{i=1}^{N} \psi_{r, i}^{2}(k) .
\end{aligned}
$$

The values of $\psi_{d, i}(k)$ and $\psi_{r, i}(k)$ for the $i$ th particle are given by

$$
\begin{aligned}
& \psi_{d, i}(k)=\frac{1}{M} \sum_{m=1}^{M} \frac{\left(\vec{r}_{i}-\vec{r}_{m}\right) \cdot\left[\vec{A}_{i}(k)-\vec{A}_{m}(k)\right]}{\left|\vec{r}_{i}-\vec{r}_{m}\right|^{2}}, \\
& \psi_{r, i}(k)=\frac{1}{M} \sum_{m=1}^{M} \frac{\left|\left(\vec{r}_{i}-\vec{r}_{m}\right)\left[\vec{A}_{i}(k)-\vec{A}_{m}(k)\right]\right|}{\left|\vec{r}_{i}-\vec{r}_{m}\right|^{2}},
\end{aligned}
$$

where $m$ is the index and $M$ the number of neighboring particles of particle $i, \vec{r}_{m}$ is the positional coordinate of a neighboring particle, and $\vec{A}_{i}(k)$ is the eigenvector of particle $i$ for mode $k$.

In Figs. 8(c) and 8(d) we plot $\psi_{d}(k)$ (solid line) and $\psi_{r}(k)$ (dotted line) as a function of frequency for clusters with $N$ $=200$, confined by $Z=200$ and $Z=4000$. The frequency dependence of the rotor $\psi_{r}(k)$ and the divergence $\psi_{d}(k)$ depend on the ratio $N / Z$. In the STC regime there is a clear separation in frequency space between $\psi_{r}(k)$ and $\psi_{d}(k)$. The shearlike modes are excited in the low frequency region, while the compression-like modes are activated in the second-half of the spectrum. In the SFC regime, the divergence behaves very differently. The quantity $\psi_{d}(k)$ increases 
monotonically as a function of frequency, and it does not exhibit a plateau like region as in Fig. 8(d). Again, the rotor, i.e., the shearlike mode, is appreciably different from zero only in the low frequency region. From Fig. 7 we note that, in the SFC regime, the shearlike modes are more related to excitations at the border of the cluster, while the compressionlike modes mainly excite particles in the internal part of the system.

\section{The magnetic field dependence}

As is already known, the magnetic field does not change the energy of a classical system of charged particles, and consequently also not the structure of the 2D classical system of charges. This is a consequence of the nature of the magnetic force, which only acts on moving particles. On the other hand, the motion of charged particles in a magnetic field is significantly affected, and this is directly reflected on the normal modes of the system. We followed Ref. [28] to calculate the normal modes of the system in the presence of a perpendicular magnetic field. To obtain the normal modes of a finite system in this case, we assumed an oscillatory solution of each particle around its equilibrium position, namely, $\quad \vec{A}_{m}(t)=\operatorname{Re}\left[\vec{R}_{m} e^{i \omega t}\right]$, for every particle $m$ $=1, \ldots, N$. The resulting equations of motion for the $x, y$ coordinates can be summarized in the following expression:

$$
\left(\omega^{2} \delta_{\alpha \beta, n m}-H_{\alpha \beta, n m}+i \omega \omega_{c} \varepsilon_{\alpha \beta z} \delta_{n m}\right) R_{\beta, m}=0,
$$

where $\alpha, \beta=x, y, n, m=1,2, \ldots, N, R_{\beta, m}$ is the particle displacement from its equilibrium position, $\varepsilon_{\alpha \beta z}$ is the Levicivita tensor, $\delta_{\alpha \beta, n m}$ and $\delta_{n m}$ are the Kronecker deltas, $\omega_{c}$ the cyclotron frequency, $i=\sqrt{-1}$, and $\omega$ the frequencies of the normal modes. Due to the presence of the magnetic field the set of Eqs. (9) have complex solutions. As a consequence, the eigenvectors have an imaginary component indicating that the response of the system is no longer in phase with an applied oscillating electric field, and each particle performs a rotational motion $\vec{u}_{m}(t)=\operatorname{Re}\left[\vec{R}_{m}\right] \cos \omega t$ $-\operatorname{Im}\left[\vec{R}_{m}\right] \sin \omega t$. Differently from the zero magnetic field case, we have now to plot the real and imaginary parts of the eigenvectors. As a reference case, we mention here the solution of the simple problem of a charged particle confined by a parabolic potential moving in a plane, and in the presence of a perpendicular uniform magnetic field. The normal modes of the particle correspond to an oscillatory motion around its equilibrium position in a circular trajectory. It is important to emphasize that the real and imaginary components of the eigenvector are perpendicular to each other, and of equal magnitude in this case, indicating that they have a difference of phase equal to $(2 n+1) \pi / 2(n=0,1,2,3, \ldots)$. We will see that in a many-body system this is no longer the case.

In Fig. 9 we show a typical spectrum as a function of the cyclotron frequency $\omega_{c}$ obtained for clusters with $N=10$ and $N=50(Z=50)$ electrons. The frequencies are split into two sets or bands with the same number of modes, which we call now "up" band, in which the frequencies follow the cyclotron frequency (dotted line), and "down" band, in which the
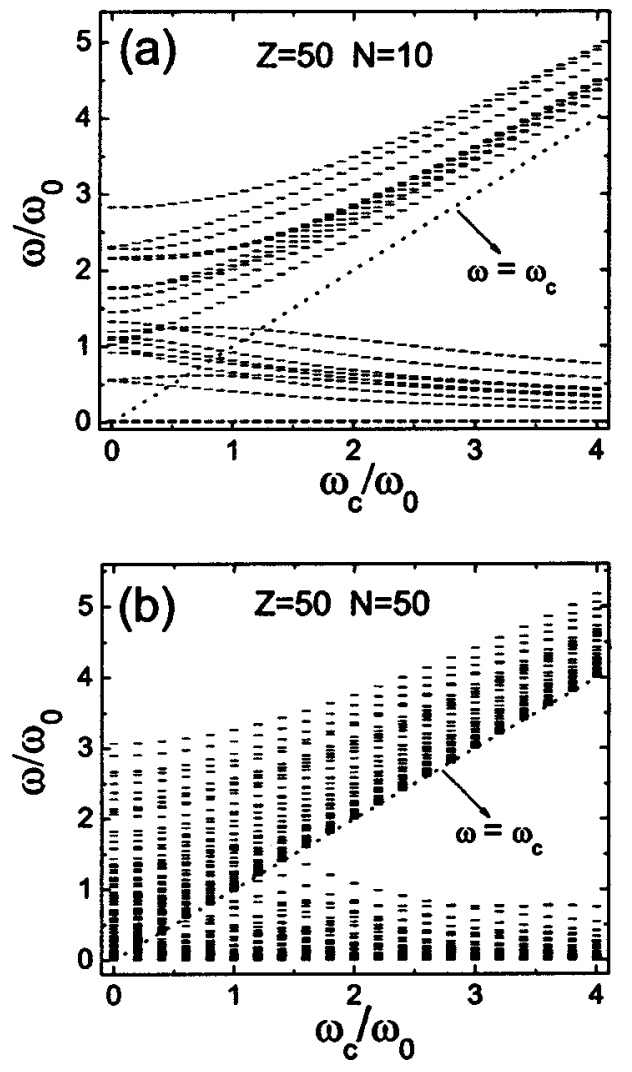

FIG. 9. Frequencies of the normal modes as a function of the cyclotron frequency. The number of particles $N$ and the confinement charge $Z$ are indicated in the figure.

value of the frequencies decreases with the cyclotron frequency. In spite of the reduced number of particles, this behavior is in qualitative agreement with the experimentally observed spectrum of a gas of electrons (density $\approx 10^{8} \mathrm{~cm}^{-2}$ ) trapped in surface states above a liquid-helium surface [29]. The nonzero width of the two-branch spectrum is not observed in an infinite system, and this effect is a consequence of the finite size of the cluster. The lower branch of frequencies was associated with the edge magnetoplasmons. In our finite cluster we found that some excitation modes are characterized by the circular motion of all particles around their respective equilibrium positions with uniform velocity. This case is characterized by the real and the imaginary components of eigenvectors perpendicular to each other, and with the same magnitude. In another kind of excitation mode, only some particles in the cluster have a circular uniform motion, while the other ones have there real and imaginary components nonperpendicular to each other, indicating that the particles move in a noncircular trajectory around their respective equilibrium positions. In order to illustrate these modes, we show in Fig. 10 the real (thick arrows) and imaginary (thin arrows) components of the eigenvectors field for the cluster $N=3(Z=50)$ in the presence of a uniform magnetic field with $\omega_{c} / \omega_{0}=2.0$. The modes $k$ $=1,2,3$ are in the lower band, while the modes $k=4,5,6$ are in the upper band (see Fig. 12). In this case $(N=3, Z$ $=50$ ) we noticed that the modes $k=2,3,4,5$ have the real and the imaginary components of the eigenvectors already per- 

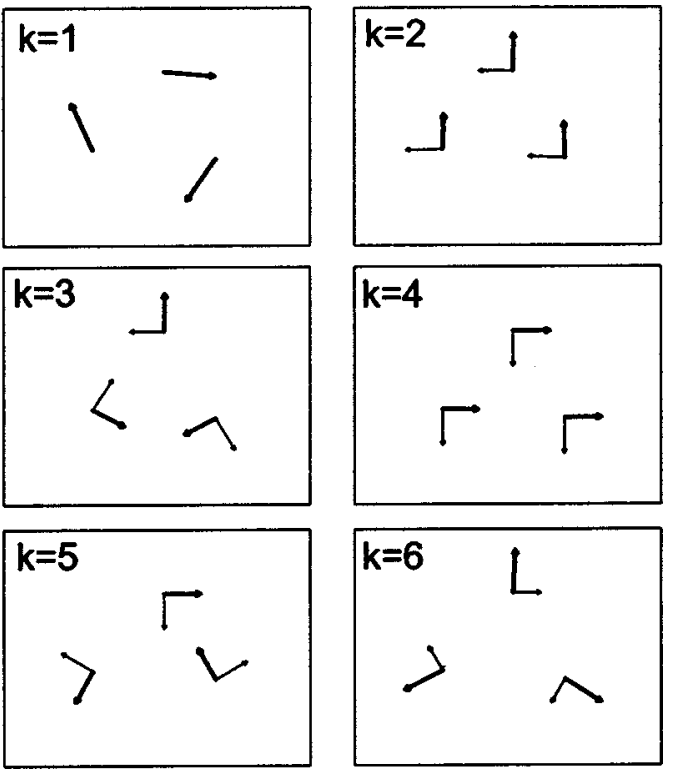

FIG. 10. Real (thick arrows) and imaginary (thin arrows) components of the eigenvectors for the cluster $N=3, Z=50$ in the presence of a perpendicular magnetic field $\omega_{c} / \omega_{0}=2$.

pendicular to each other, and of equal magnitude (the mode $k=1, \omega / \omega_{0}=0$, does not have imaginary component). For lager values of $\omega_{c}$ the mode $k=6$ also behaves like the other modes, indicating the circular uniform motion of the particles. In other words, for a sufficiently high value of the magnetic field the normal modes correspond to a circular uniform motion of the particles around their respective equilibrium positions.

In order to characterize the change of the nature of the modes in the presence of a magnetic field, we resort to the calculation of the divergence $\psi_{d}(k)$ and the rotor $\psi_{r}(k)$ of
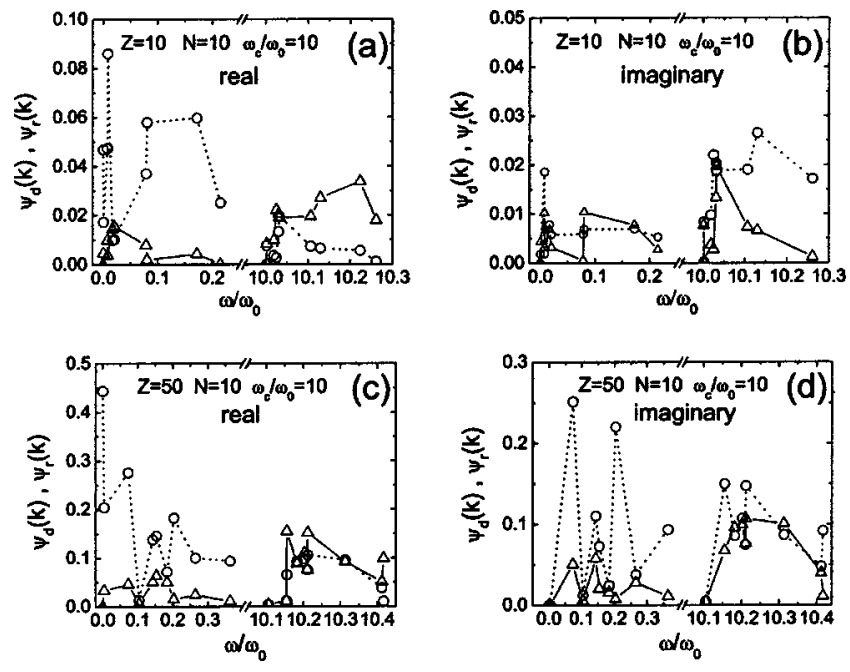

FIG. 11. The divergence $\psi_{d}$ and the rotor $\psi_{r}$ of the real and imaginary components of the eigenvectors field for clusters with $N=10$ and (a) $Z=10$-real component, (b) $Z=10$-imaginary component, (c) $Z=50$-real component, (d) $Z=50$-imaginary component, in the presence of an axial magnetic field $\omega_{c} / \omega_{0}$ $=10$.

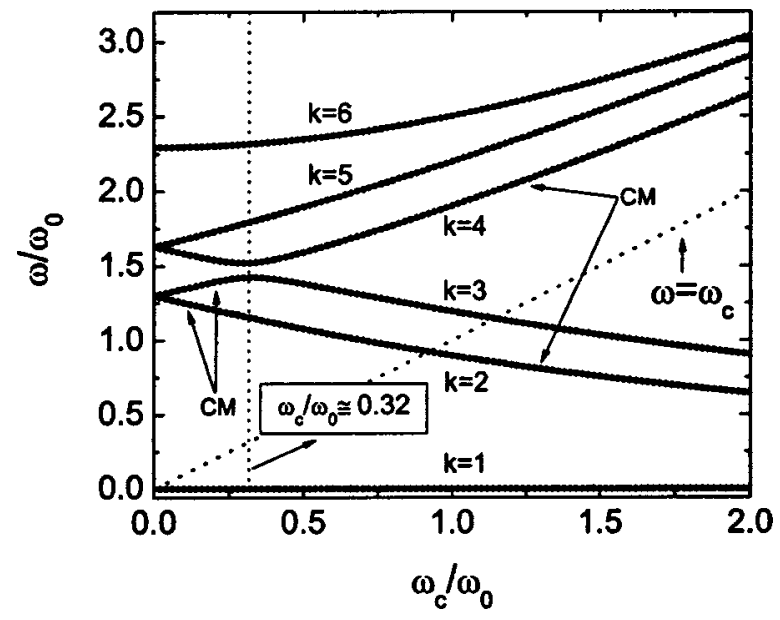

FIG. 12. Frequencies of the normal modes as a function of the cyclotron frequency for the cluster with $N=3$ and confinement charge $Z=50$. The numbers indicating each mode are presented in the figure.

the eigenvectors' field. In the zero magnetic field regime, we found that in the small frequency limit the modes are mostly shearlike in character, i.e., large value for $\psi_{r}(k)$, while in the high frequency limit they are mainly compressional, i.e., large value for $\psi_{d}(k)$ [Figs. 8(c) and 8(d)]. This behavior is valid in both regimes of confinement (STC and SFC). In Fig. 11 the quantities $\psi_{d}(k)$ and $\psi_{r}(k)$ (for the real and the imaginary components of the eigenvectors' field) are presented as a function of frequency for clusters with $N=10(Z=10,50)$ electrons under nonzero magnetic field $\left(\omega_{c} / \omega_{0}=10\right)$. Independently of the confinement regime, we notice that the rotor is substantially different from zero in the high frequency region of each band, which is in contrast to the zero magnetic field case. The divergence also presents a different behavior since it is smaller than the rotor in the high frequency limit of the low frequency band. Thus the nature of the modes is essentially modified by the magnetic field which introduces a clear rotational component to the motion of the particles, which as a consequence enhances $\psi_{r}(k)$.

In Fig. 12 the frequencies of the normal modes for the cluster $N=3(Z=50)$ are presented as a function of the cyclotron frequency. For $\omega_{c} / \omega_{0} \lesssim 0.32$ the excitation of the center of mass corresponds to the modes $k=2$ and $k=3$. For the value of the magnetic field $\omega_{c} / \omega_{0} \approx 0.32$ there is an anticrossing, and the modes are changed such that the excitation of the center of mass now corresponds to the modes $k$ $=2$ and $k=4$. As can be observed in Fig. 12, there is no crossing between the modes $k=3$ and $k=4$, but a modification of the character of each of the excitation modes occurs. For larger values of the confinement charge $Z$, the gap between the modes (around $\omega_{c} / \omega_{0} \approx 0.32$ ) $k=3$ and $k=4$ becomes very small. On the other hand, for smaller $Z$ the gap between the modes $k=3$ and $k=4$ increases, which is indicative of a stronger interaction between the two modes, and which is a consequence of the increased importance of the nonparabolic nature of the confinement potential.

The real and the imaginary parts of the eigenvectors are not always directed perpendicular to each other, as already 

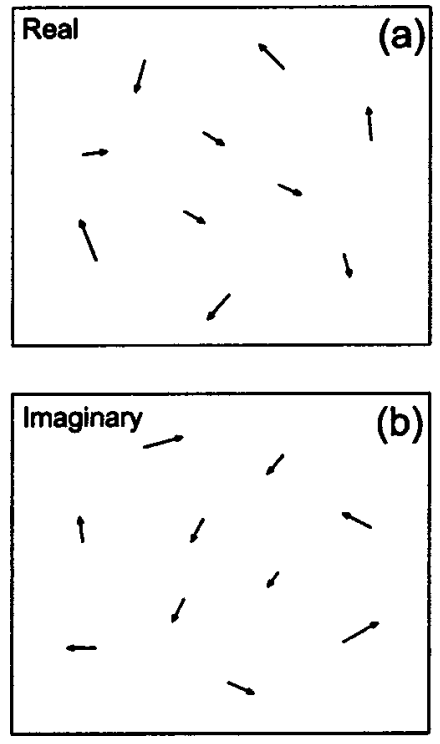

FIG. 13. Real (a) and imaginary (b) components of the eigenvectors for the mode $k=3$ of the cluster $N=10, Z=50$ in the presence of a perpendicular magnetic field $\omega_{c} / \omega_{0}=1.6$.

commented. An example of such a behavior is illustrated in Fig. 13, where the real and imaginary components of the eigenvectors for the cluster $N=10(Z=50)$ (in the presence of a magnetic field $\left.\omega_{c} / \omega_{0}=1.6\right)$ are presented for the mode $k=3$. As can be observed both components exhibit a vortexantivortex pair excitation which are $90^{\circ}$ rotated with respect to each other. Most of the real-imaginary components of the eigenvectors are not perpendicular to each other, and also they do not have the same magnitude. These features indicate that the particles have rotational motion, but with a nonuniform velocity.

As was shown in Sec. III B, in the SFC regime $(N \approx Z)$ and for the zero magnetic field case, there is a high concentration of modes with small frequencies. A similar distribution of modes was also observed in the nonzero magnetic field case. However, the interesting feature here is that the distribution of modes, like the one in the zero magnetic field case, occurs in each band, as can be clearly seen in Fig. 9(b). Most of the modes are concentrated in the lower part of both the upper and the lower bands. In the STC regime the distribution of frequencies in each band is like that presented in the zero magnetic field case [Fig. 2-region (VI)]. We also observed that in the STC regime the coupling between the frequencies in the upper band with the cyclotron frequency occurs for higher values of the magnetic field than in the SFC case.

In the STC regime, where the system behaves like a parabolic confined cluster, a c.m. mode is observed. We notice that under an applied magnetic field the degenerated c.m. mode is split due to the fact that the magnetic field couples the $x$ - and $y$ directions. One of the c.m. modes is observed in the lower band, while the other one in the upper band. This is a consequence of the coupling between the restoring force due to the positive charge and the magnetic force. When the magnetic force is in the same direction of the electric force, the total restoring force is large, leading to the upper frequency. On the other hand, when the forces are in opposite directions, the resulting restoring force is small leading to a frequency which diminishes with increasing magnetic field, i.e., it behaves as $1 / \omega_{c}$ for large $\omega_{c}$.

\section{CONCLUSIONS}

We studied numerically the normal mode spectra of a two-dimensional classical system consisting of $N$ negative charged particles bounded by a Coulomb potential. The $N$ negative particles are kept together by a positive charge $Z$ located at a distance from the plane where the electrons are allowed to move. The normal mode frequencies were calculated numerically as a function of $Z$ and $N$. Depending on the ratio $N / Z$ different distributions of modes were observed. An analysis of the lowest normal mode frequency showed that in the limit $N / Z \ll 1$, the experimental results observed in dusty plasmas are very similar to the results found in the present system. The normal modes observed in our pure Coulomb system and those in the screened Coulomb system under parabolic confinement are obtained by tuning the value of the positive charge $Z$.

The normal mode spectrum in the presence of a perpendicular magnetic field was also calculated. The frequencies of the normal modes are strongly dependent on the magnetic field intensity and on the ratio $N / Z$. The presence of some modes with frequencies that decrease with increasing magnetic field is in agreement with the resonant spectra observed in an electron gas above a liquid-helium surface, in spite of the reduced number of particles in the present system. The "shearlike" character of the modes is increased with increasing value of the magnetic field.

\section{ACKNOWLEDGMENTS}

W.P.F. and G.A.F. were supported by the Brazilian National Research Councils (CNPq, CAPES), and the Ministry of Planning (FINEP). Fruitful discussions with A. Vagov and B. Partoens are gratefully acknowledged. Part of this work was supported by the Flemish Science Foundation (FWOV1), the "Onderzoeksraad van de Universiteit Antwerpen" (GOA), and the EU-RTN network on "Surface electrons."
[1] L. Jacak, P. Hawrylak, and A. Wójs, Quantum Dots (SpringerVerlag, Berlin, 1998).

[2] R.S. Crandall and R. Williams, Phys. Lett. A 34, 404 (1971).

[3] C.C. Grimes and G. Adams, Phys. Rev. Lett. 42, 795 (1979).
[4] M. Golosovsky, Y. Saado, and D. Davidov, Phys. Rev. E 65, 061405 (2002).

[5] J.H. Chu and L. I, Phys. Rev. Lett. 72, 4009 (1994).

[6] V.M. Bedanov and F.M. Peeters, Phys. Rev. B 49, 2667 (1994). 
[7] V.A. Schweigert and F.M. Peeters, Phys. Rev. B 51, 7700 (1995).

[8] A. Melzer, Phys. Rev. E 67, 016411 (2003).

[9] M. Klindworth, A. Melzer, A. Piel, and V.A. Schweigert, Phys. Rev. B 61, 8404 (2000).

[10] A. Melzer, M. Klindworth, and A. Piel, Phys. Rev. Lett. 87, 115002 (2001).

[11] M. Kong, B. Partoens, and F.M. Peeters, New J. Phys. 5, 23 (2003).

[12] G.A. Farias and F.M. Peeters, Solid State Commun. 100, 711 (1996).

[13] W.P. Ferreira, G.A. Farias, H.A. Carmona, and F.M. Peeters, Solid State Commun. 122, 665 (2002).

[14] R. Bubeck, C. Bechinger, S. Neser, and P. Leiderer, Phys. Rev. Lett. 82, 3364 (1999).

[15] K. Zahn, R. Lenke, and G. Maret, Phys. Rev. Lett. 82, 2721 (1999).

[16] Weijia Wen, Lingyun Zhang, and Ping Sheng, Phys. Rev. Lett. 85, 5464 (2000).

[17] I. Schweigert, V.A. Schweigert, and F.M. Peeters, Phys. Rev. Lett. 84, 4381 (2000).

[18] U. Konopka, D. Samsonov, A.V. Ivlev, J. Goree, and V. Stein- berg, Phys. Rev. E 61, 1890 (2000).

[19] F. Cheung, A. Samarian, and B. James, New J. Phys. 5, 75 (2003).

[20] O. Ishihara, T. Kamimura, K.I. Hirose, and N. Sato, Phys. Rev. E 66, 046406 (2002).

[21] W.P. Ferreira, A. Matulis, G.A. Farias, and F.M. Peeters, Phys. Rev. E 67, 046601 (2003).

[22] N. Metropolis, A.W. Rosenbluth, M.N. Rosenbluth, A.M. Teller, and E. Teller, J. Chem. Phys. 21, 1087 (1953).

[23] L. Bonsall and A.A. Maradudin, Phys. Rev. B 15, 1959 (1977).

[24] R.S. Crandall, Phys. Rev. B 8, 2136 (1973).

[25] Notice that in regions (IV), (V), and (VI) the Coulomb confinement system is in the STC regime and the Coulomb confinement potential may be approximated by a parabolic one.

[26] In the case $N=50$ and $Z=10000$ the maximum radius is $R_{\text {max }} \approx 0.18$, while for the case $N=50$ and $Z=50$ we found $R_{\text {max }} \approx 7.92$.

[27] We investigated numerically clusters with $N$ up to 200 .

[28] V.A. Schweigert and F.M. Peeters, J. Phys.: Condens. Matter 10, 2417 (1998).

[29] D.B. Mast, A.J. Dahm, and A.L. Fetter, Phys. Rev. Lett. 54, 1706 (1985). 